\title{
Distortions of perceived visual directions out of pictures
}

\author{
STEPHEN R. ELLIS \\ NASA Ames Research Center, Moffett Field, California \\ and University of California, Berkeley, California \\ STEPHEN SMITH \\ California State University, San Jose, San Jose, California \\ and \\ MICHAEL W. MCGREEVY \\ NASA Ames Research Center, Moffett Field, California
}

\begin{abstract}
Two experiments are reported examining judgments from 16 subjects who indicated the apparent direction of a photographed pointer that was rotated to different physical positions while being photographed. The photographs themselves were rotated about a vertical axis to several positions with respect to the subjects' central viewing axis. The results replicate the well-known distortion in apparent direction associated with photographed pointers positioned to project directly out of the plane of the photograph. This effect has been described by Goldstein (1979) as the "differential rotation effect" because its magnitude is reduced as the depicted angle of the pointer becomes less orthogonal to the photograph. Analysis of the two-dimensional properties of the projected images shows that this differential rotation is related to projected angles on the surface of the photograph. This analysis may explain why circular objects often do not appear to be correctly drawn in the periphery of geometrically correct projections.
\end{abstract}

A picture that is intended to accurately represent objects in three-dimensional space is inherently ambiguous because it exists as a two-dimensional object in its viewer's visual space. However, it also represents an alternative or virtual space that is defined by the parameters of the geometric projection used to produce and view it (McGreevy \& Ellis, in press; Rosinski \& Farber, 1980) and by the viewer's assumptions concerning depicted relationships among displayed objects (Attneave, 1972; Negrin \& Grunwald, 1985). In the case of a perspective projection, the discrepancy between picture and virtual space can be reduced by observing the picture from the position in which it was constructed. It can be completely resolved only by viewing it monocularly through a small pupil without head or eye movements. However, observers usually are not at the center of projection and can only be positioned there by a combination of rotations and translations. Their usual view away from the center of projection might be expected to result in systematic geometric distortion in the shapes, distances, and lines represented by the photograph.

We would like to thank Marcia Brooks for help in collecting some of the data and for assisting in preliminary experiments; Arnold Stoper, Malcolm Cohen, Mary Kaiser, and Roger Remington for helpful discussions concerning analysis of the experimental data; and Arthur Grunwald, John Perrone, and Kenneth Nielsen for critically reading the manuscript. Please send reprint requests to Stephen R. Ellis, NASA Ames Research Center, MS 239-3, Moffett Field, CA 94035
One way in which these distortions can manifest themselves is by causing observers to inappropriately perceive the size and shape constancy of the objects represented in the picture. The constancies that help the observer maintain a stable perception of the picture plane while moving relative to the picture are not appropriate, however, for the objects represented in the picture. These objects are depicted at a variety of different physical depths and orientations, but their images are located on the surface of the picture. (Wallach, 1985; Wallach, Stanton, \& Becker, 1974).

Accordingly, one would expect observers to perceive substantial apparent distortion of the image in a picture when moving away from its center of projection. (Significantly, these distortions are rarely noted in pictures intended to accurately represent a scene.) Pirenne (1970, 1975) argued that the reason for the apparent constancy of the perception of the image is that the observer develops a "subsidiary awareness" of the plane of the picture itself. This awareness, which may be derived from binocular and other cues to the position of the surface of the photograph, allows the observer to compensate for the unusual viewing position (Rosinski, Mulholland, Degelman, \& Farber, 1980; cf. Bengston, Stergios, Ward, \& Jester, 1980).

There are, however, certain conditions that exaggerate these distortions. A good example of such a condition is a photograph of a scene which itself contains 
another photograph. In this situation the distortion caused by viewing the photographed photograph from a point away from its center of projection is striking because the viewer has no physical cues, such as accommodative or vergence demand, to its position in space (Pirenne, 1970). In general, the amount of distortion may be related to the degree to which the observer is unable to sense the actual position of the plane of the picture in which an image is presented.

The distortions of ordinary perspective pictures are also particularly prominent under certain specific orientations of the depicted objects. When an object is depicted to extend orthogonally out of the plane of a picture, it is commonly seen by a moving observer to also move and to continue to point towards the observer as he/she moves laterally away from a centered frontal view of the photograph. A good example of this is Uncle Sam's finger pointing out of the World War I U.S. Army recruiting poster. This phenomenon was studied by Goldstein (1979), who called it the "differential rotation effect" because it varies with the angle at which objects are depicted to project out of the plane of the picture. It is strongest for objects projecting $90^{\circ}$ out of the plane of the photograph and weakest for those projecting parallel to the plane of the picture.

One may interpret the apparent rotation of the pointing object or pointer as a case of inappropriate shape constancy. As the observer moves parallel to the face of the picture, the picture plane rotates with respect to the direction of the observer's gaze. Because the observer is aware of his/her movement, he/she expects to be able to see more of the side of the pointer, which would occur with an actual pointer. Because it does not occur with a pictured pointer, the observer perceives the pointer as continuing to point in the same direction with respect to the observer, regardless of his/her actual viewpoint. Hence, it appears to rotate with the observer as he/she moves.

The differential apparent rotation of a pointer occurs in a sequence of pictures in which the depicted azimuth angle $(\alpha)$ of a pictured pointer varies with respect to the plane of the picture itself. The variation in apparent rotation may be interpreted as being due to the changing length of the projection of the pointer on the picture plane. At the observer's eye level, a pointer that is orthogonal to the picture plane projects approximately to a single point, whereas other orientations project to line segments. As the picture itself is rotated with respect to the observer, the pointer provides almost no information as to its surface orientation on the picture plane. Therefore, the pointer is most subject to apparent rotation as the picture is rotated with respect to the observer. In contrast, when a pointer of length $l$ has a projected length on the picture surface $\left(l_{s}\right)$ where $l_{s}=l \cos (\alpha)$, this projection provides information about actual orientation of the image of the pointer in the picture surface. This information is provided by a secondary projection of the picture onto the frontal plane of a viewer as described in Equation 1,

$$
l_{f}=\frac{l_{s}}{m_{c}} \cos (\alpha) \cos (v),
$$

where the projected length $\left(l_{f}\right)$ is that of a pointer's image on the frontal plane a unit distance from a viewer. This equation represents a pointer positioned directly in front of a viewer at eye level and pivoted with no elevation angle. It shows that the projected length is a function of the rotation $(v)$ of the photograph about its central vertical axis. In this equation, $m_{c}$ is a magnification factor dependent upon the viewing distances used to create and view the projection. (Appendix A provides a more complete discussion of the relevant projective geometry.) Accordingly, $l_{f}$ provides a geometric basis for the hypothesis that the apparent rotation of the pointer, later called total rotation, should be a cosine function of depicted azimuth $\alpha$ and, thus, that the pointer should appear to rotate differentially for different depicted azimuth angles.

The occurrence of this differential rotation effect requires the observer to interpret the images in the picture as representing objects in a virtual space. To the extent that the images are seen merely as marks on the surface of the picture, all azimuthal judgments of the orientation of those objects can only be in the plane of the picture. Therefore, since the picture itself should exhibit constancy of shape, the marks on its surface should not appear to rotate as the observer moves.

In the first of the following two experiments, we applied systematic measurements of the differential rotation effect to photographs with full pictorial cues containing pointers of systematically varied orientation. The second experiment demonstrated a new psychophysical influence that modulates the effect when it is produced with these stimuli. In both experiments, we used the technique, suggested by Goldstein (1979), of rotating the photograph with respect to a stationary observer rather than moving the observer parallel to the plane of the photograph while the observer centers his/her gaze on it. Although the selected technique is not completely identical to actually displacing the observer, Goldstein's results show it to be adequate to demonstrate the desired phenomenon.

\section{EXPERIMENT 1}

\section{Method}

Stimuli. To produce a set of realistic pictorial stimuli, a series of photographs was taken of a pointer attached to the top of a photographic tripod that was positioned in a corner of our laboratory. As shown in Figure 1, rectilinear contours, shadows, and room clutter would provide monocular cues to the position and orientation of the pointer in the room.

A standard $35 \mathrm{~mm}$ camera with a $50 \mathrm{~mm}$ lens was used. The camera was leveled, centered, and pointed at the top of the tripod. The tripod was then adjusted to be horizontal with respect to the floor. Photographs of the pointer in a series of positions were taken as it was rotated horizontally in $30^{\circ}$ increments from a position pointing right and parallel to the film plane through a position pointing toward the camera to a final position pointing left and $180^{\circ}$ 


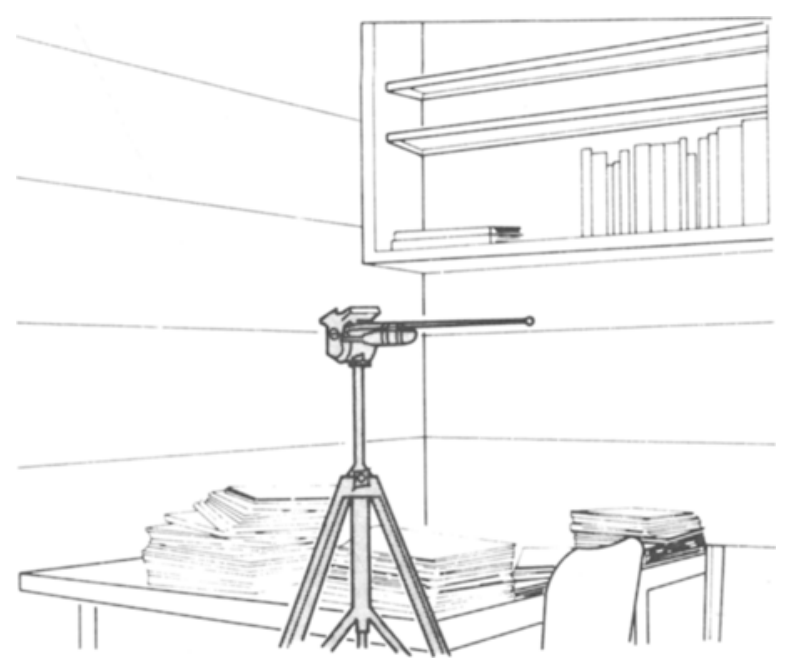

Figure 1. A sketch made from a photograph used in Experiment 1, depicting a pointer at an angle of $30^{\circ}$ with the plane of the photograph.

opposed to the initial direction. In terms of Goldstein's (1979) nomenclature, these angles are the "depicted angles" of the pointer. Prints of the photographs were made and the resulting center of perspective projection was centered $34 \mathrm{~cm}$ in front of the center of the photograph.

The photographs were glued on cardboard and mounted on a modified $13 \times 30 \mathrm{~cm}$ clipboard, which could be rotated through $180^{\circ}$ about a vertical axis. These rotations allowed the presentation of the photographs at a variety of angles with respect to the subject's line of sight. In keeping with Goldstein's (1979) nomenclature, these angles are the "viewing angles," and they are measured counterclockwise with respect to the line of sight.

Immediately above the clipboard was an adjustable $12-\mathrm{cm}$ pointer which rotated about the same center as the clipboard. The center of rotation of the pointer was $7 \mathrm{~cm}$ above the center of the photograph mounted on the clipboard. The pointer was driven by a reversible ac motor controlled by a center-off momentary-contract threeposition switch and rotated either clockwise or counterclockwise at about $4 \mathrm{rpm}$. This pointer allowed subjects to indicate their perceived orientation of the pointer depicted in each photograph. A positive perceived angle is measured clockwise with respect to the plane of the photograph. A negative perceived angle is measured counterclockwise with respect to the photograph. Figure 2 illustrates these definitions with several examples. The subject's perceived angle was read off a $360^{\circ}$ protractor that was attached at the center of rotation. Perceived angles could be read to about $\pm 1^{\circ}$ accuracy. Viewing angles, which were read off a separate protractor, could be measured to about $\pm 2.5^{\circ}$ accuracy due to the thickness of the clipboard and some uncertainty about its actual center of rotation.

Experimental conditions and design. The experimental apparatus was placed on the end of a table in a photopically illuminated laboratory room. The subject sat in a chair and viewed the photographs of the pointer and tripod binocularly across the table from a distance of $120 \mathrm{~cm}$. At this distance, when the viewing angle was $90^{\circ}$ and the depicted angle was $0^{\circ}$, the image of the pointer on the picture surface extended $8 \mathrm{~cm}$ and subtended a visual angle of $3.8^{\circ}$. Changing the depicted angle of the pointer to $90^{\circ}$ exposed only its width, which was about $0.25 \mathrm{~cm}$. The viewing position, behind the center of projection, minified the images on the picture plane by a constant factor of about 3.5:1. Because of the small angular extent of the pointer image, this minification resulted in small angular distortions in the lines of sight to the image of less than $10^{\circ}$ in the worst case. The projected length and subtended angle were changed when either the depicted or viewing angle changed according to Equation 1. Binocular viewing was used to enhance the subject's awareness of the plane of the photograph for possible subsequent manipulation. As Goldstein (1979) noted, the differential rotation effect was "essentially the same for monocular and binocular viewing." 'The subject's head was positioned with a head- and chinrest so as to align his midline with the pictured top of the tripod, which was centered in the photograph. His eyes were level with the center of the photograph.

The subject's task was to align the adjustable pointer above the photograph to point in the same direction as the pointer depicted in the photograph. Initially, the pointer was randomly placed in either of the positions orthogonal to the subject's line of sight.

We measured the differential rotation effect, using what Goldstein (1979) termed the "total rotation" for a pointer depicted at one angle. The total rotation is defined as the change in the perceived direction of the pointer when the viewing angle is rotated through $180^{\circ}$. Maximum total rotation occurs, for example, for a $90^{\circ}$ depicted angle, such as Uncle Sam's finger in the recruiting poster. In this case, perceived angle approximately equals viewing angle. The main focus of our first experiment was to examine the effect with binocularly viewed and pictorially complete stimuli; hence, we did not attempt a complete replication of Goldstein's experiment.

In the course of testing the apparatus it became apparent that subjects would have to be carefully instructed to indicate a perceived angle coming out of the picture. They would also have to be familiarized with the range of depicted positions. Because of the spatial ambiguity caused by viewing one pointer rotated out of the picture plane and another rotated into the picture plane at the same angle, it was possible to confuse the two. All of our depicted angles came out of the picture plane toward the subjects, and they were carefully instructed in this fact before they began the experiment.

The first experiment was conducted as a fully crossed design with viewing angle and depicted angle as independent variables and with perceived orientation as the dependent variable. A repeated measures design was used on 8 subjects. Viewing angle was set to $15^{\circ}$, $45^{\circ}, 70^{\circ}, 90^{\circ}, 110^{\circ}, 135^{\circ}$, and $165^{\circ}$, and the depicted angle was set to $0^{\circ}, 30^{\circ}, 60^{\circ}, 90^{\circ}, 120^{\circ}, 150^{\circ}$, and $180^{\circ}$. Separate randomized sequences were prepared in order to present each subject with the $\mathbf{4 9}$ distinct conditions.

Subjects. Eight paid subjects participated in the experiment. They were selected from the subject pool used by the Aerospace Human Factors Division at the NASA Ames Research Center and had no particular qualifications other than normal vision with or without glasses

\section{Results}

An analysis of variance (ANOVA) on perceived orientation showed that viewing angle $[F(6,42)=36.9$, $p<.001]$ and depicted angle $[F(6,42)=316.8$, $p<.001]$ were statistically significant, as was their interaction $[F(36,252)=1.512, p<.037]$. The means of the interaction, which are plotted in Figure 3, show that the differential rotation effect is obtained with our pictorially complete stimuli. This effect, indicated by the statistically significant interaction, is shown in Figure 3 by the decreasing slope of the lines as the parameter of each line-the depicted angle-departs from $90^{\circ}$. As the depicted angle changes from $0^{\circ}$ to $180^{\circ}$ in $30^{\circ}$ increments, the slopes of the perceived orientations shown in Figure 3 are, respectively, $0.60,0.61,0.66,0.87,0.65,0.61$, and 0.58 . Thus, the total rotation of the perceived orientation of the pointer in the photograph is correspondingly 

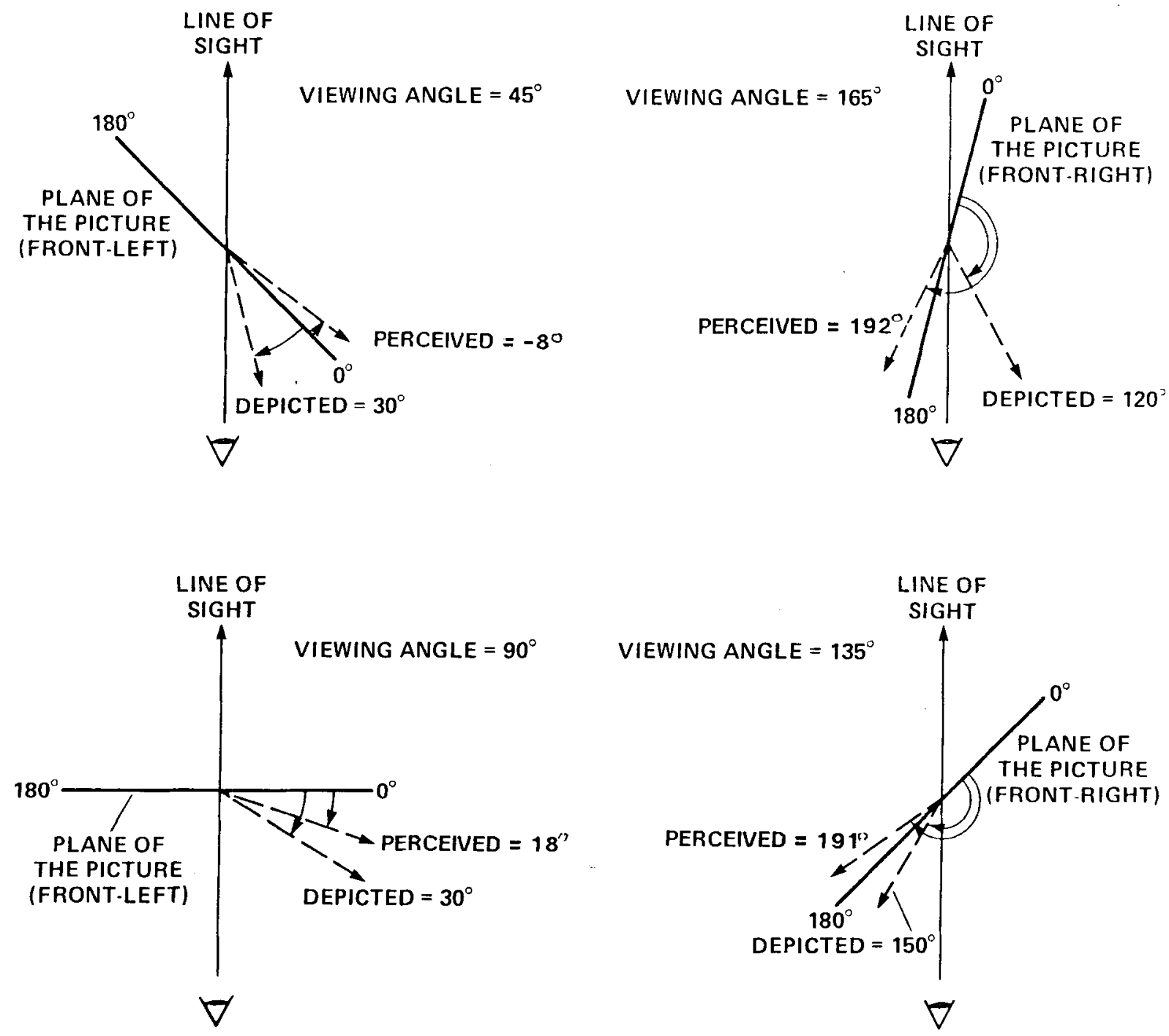

Figure 2. Several examples of different viewing angles with various depicted angles and the corresponding across-subject mean perceived angles from Experiment 1 . The examples are displayed as if viewed from above the apparatus and looking down along the axis of picture plane rotation.

reduced. For example, the total rotation of the $90^{\circ}$ depicted angle was $125^{\circ}$, that is, a maximum perceived angle of $150^{\circ}$ minus a minimum perceived angle of $25^{\circ}$ (see Figure 3). In contrast, total rotation of $0^{\circ}$ and $180^{\circ}$ was $94^{\circ}$ and $81^{\circ}$, respectively.

\section{Discussion}

One interesting difference between Goldstein's (1979) experiment and our experiment was that he pivoted his pointer in the middle and we pivoted it at one end. Thus, we collected data from both rightward- and leftwarddirected pointers. Goldstein, in contrast, did not strongly emphasize this difference, because he used an orthogonal projection of his pointer on his presentation picture (personal communication, July 1987). Therefore, his presentation of T-shaped stimuli would appear to result in es- sentially equivalent projected directions for both clockwise and counterclockwise rotations. In both his experiment and our experiments, the stimuli were nearly orthogonal projections.

For comparison with Goldstein's (1979) data, we have collapsed data from pointers directed toward symmetrical positions, that is, $30^{\circ}$ with $150^{\circ}$ and $60^{\circ}$ with $120^{\circ}$. When the data from Figure 3 are collapsed across symmetrical positions, the results from Experiment 1 generally replicate Goldstein's findings. The slopes of the functions we measured are somewhat different, especially for the smaller depicted angles of $0^{\circ}$ and $30^{\circ}$. Our range of slopes is also a bit different. We have slopes ranging from 0.58 to 0.87 , whereas Goldstein's functions had slopes ranging from about 0.5 to 1.0 . This reduced range of slopes corresponds to the generally reduced differential 


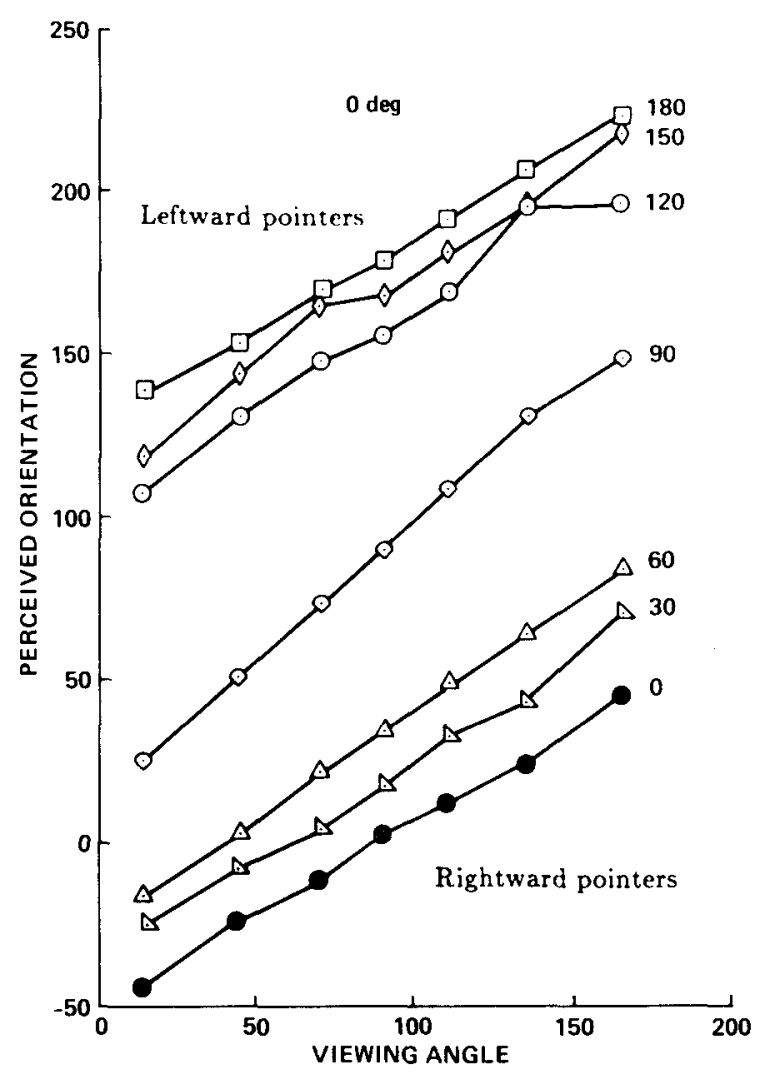

Figure 3. Mean perceived pointer orientation for viewing angles and depicted angles in Experiment 1 with a $0^{\circ}$ elevation condition. Plotted using Goldstein's conventions.

rotation of the pointer as the depicted angle changes, which is consistent with Goldstein's observation that reduction in the length of the pointer reduces the amount of differential rotation. Our use of a pointer pivoted at one end resulted in our stimuli's being a little more than half the size of the depicted pointer that Goldstein used.

One interesting aspect of both Goldstein's (1979) data and our data is that we both measured negative perceived angles for pointers depicting small acute angles with the plane of the photograph. The meaning of these negative angles at first puzzled us, but it can be clearly visualized by referring to Figure 2 . We tended to measure the largest negative perceived angles when the viewing angle became very small, that is, $15^{\circ}$. The negative perceived angles judged under this condition correspond to the viewer's inability to see the pointer as projecting out of the plane of the photograph. If the pointer were physically projecting out of the photograph, the viewer would expect to see this more clearly under the acute viewing condition than in a frontal view in which the pointer would be totally superimposed on the background of the picture itself. We observed a corresponding effect for a larger viewing angle, also illustrated in Figure 2, of pointers depicted to be close to the plane of the photograph. In this case the perceived angles were measured as larger than $180^{\circ}$ and the perceived position of the pointer was qualitatively the same, that is, behind the plane of the photograph.

An interesting initial hypothesis to be investigated in a second experiment was based on theories, discussed in the introduction, regarding the cause of differential apparent rotation. It was suggested that apparent rotation is a function of the observer's awareness of the orientation of the image of the pointer in the plane of the picture, and this awareness was thought to be proportional to the projected length of the pointer in the picture. One way to test this explanation is by manipulating the position of the pointer so as to vary the length of its projection in the plane of the picture.

\section{EXPERIMENT 2}

A simple way to control the evidence that the projection of the pointer is in the picture plane is to introduce an elevation or depression angle $(\epsilon)$ into the position of the pointer. This modification has a significant advantage: Not only does it change the projected length of the pointer, but it also produces a number of other changes that could provide alternative influences on the apparent direction of the pointer. For example, it changes the projected horizontal and vertical components of the pointer's image as well as their ratio (see Appendix A).

The most direct effect of the introduction of an elevation angle, however, is to increase the projected length of the pointer across most conditions of Experiment 1. As shown in Appendix A, this projected length $\left(l_{s}\right)$ on the picture surface is a function of both the depicted azimuth and the elevation in Equation 2. In this equation, $m$ is the magnification factor of the projection and $l$ is the length of the pointer:

$$
l_{s}=\frac{l}{m} \sqrt{(\sin (\epsilon))^{2}+(\cos (\epsilon) \cos (\alpha))^{2}} .
$$

The longer $l_{s}$, the greater would be the binocular cues to the position of the pointer's image on the picture plane. The frontal projected length $\left(l_{f}\right)$ also varies from the viewer's position as the viewing angle $(v)$ changes by a similar secondary projection. A significant aspect of using Equation 2 to infer the depicted position of the pointer in space is that for a $90^{\circ}$ depicted azimuth there is a substantial difference in $l_{s}$ for $0^{\circ}$ and $30^{\circ}$ elevation, specifically, the difference between approximately 0 and $l \sin (30) /(D-l \cos (30))$, where $D$ is the distance to pivot point $r$.

On our photographs, the projected length of the pointer depicted at $90^{\circ}$ azimuth was increased from $0.25 \mathrm{~cm}$ (its width) to $4.7 \mathrm{~cm}$ when a $30^{\circ}$ elevation was introduced. If this projected length were the primary factor used to infer the pointer's position, then one would expect a substantial difference in apparent rotation for pointers whose elevation angles were different because of the differential changes in length. 
The initial hypothesis for Experiment 2, therefore, was that the extra information provided by the projected length of the pointer when it is elevated or depressed should proportionately reduce the apparent rotation of the depicted angle of a pointer as the viewing angle is changed. More specifically, for any total apparent rotation $R_{t}$,

$$
R_{t}:: \frac{1}{l_{s}},
$$

and for a comparison of total rotation measured for the $0^{\circ}$ and $30^{\circ}$ elevation conditions in both experiments,

$$
\left(R_{t_{0}}-R_{t_{30}}\right):: \frac{\left(l_{s_{30}}-l_{s_{0}}\right)}{l_{s_{0}} l_{s_{30}}}
$$

\section{Method}

Stimuli. The photographs used in the second experiment were identical to those used in the first, except that the pointer was either elevated or depressed by $30^{\circ}$ for each of the depicted azimuth positions (see Figure 4). The depicted azimuths, camera setting, and background were identical to those of Experiment 1. The same procedures were used to present the photographs and to measure the subject's perceived angle.

Experimental conditions and design. The second experiment was conducted under the same laboratory conditions as the first, with the subject using the same method of adjustment to position the pointer to the perceived orientation of the pointer in the photograph. The alignment task was the same. In this case the subject was to indicate the azimuthal component of the depicted pointer, which now also had an elevation or depression angle of $30^{\circ}$. A positive elevation corresponded to an upward rotation and a negative elevation to a downward rotation.

The second experiment was also conducted as a fully crossed design with viewing angle and depicted angle as independent variables and with perceived orientation as the dependent variable. A repeated measures design was used, with direction of elevation added as another independent variable to those previously used. Viewing angle and depicted angle were set to the same values used in Experiment 1 . Separate randomized sequences were prepared in

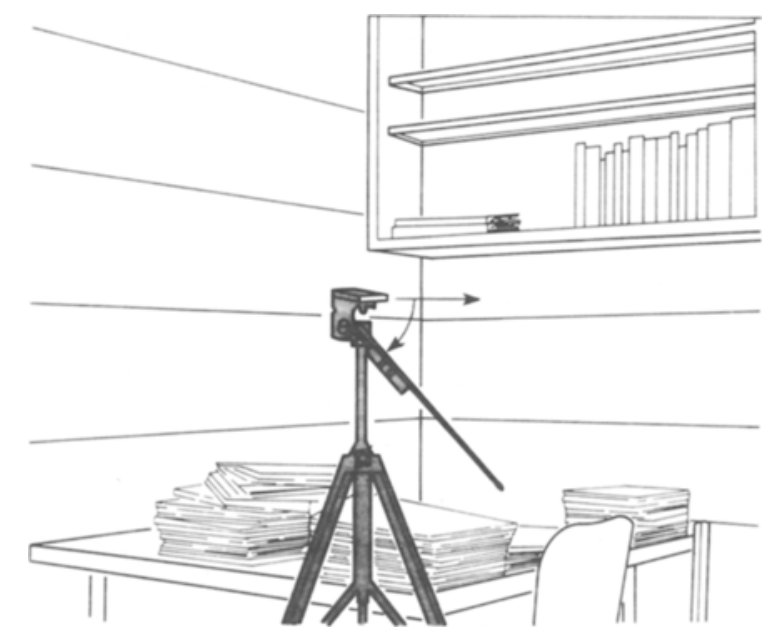

Figure 4. A sketch made from a photograph used in Experiment 2, depicting a pointer at an angle of $30^{\circ}$ and $a 30^{\circ}$ depression with the plane of the photograph.

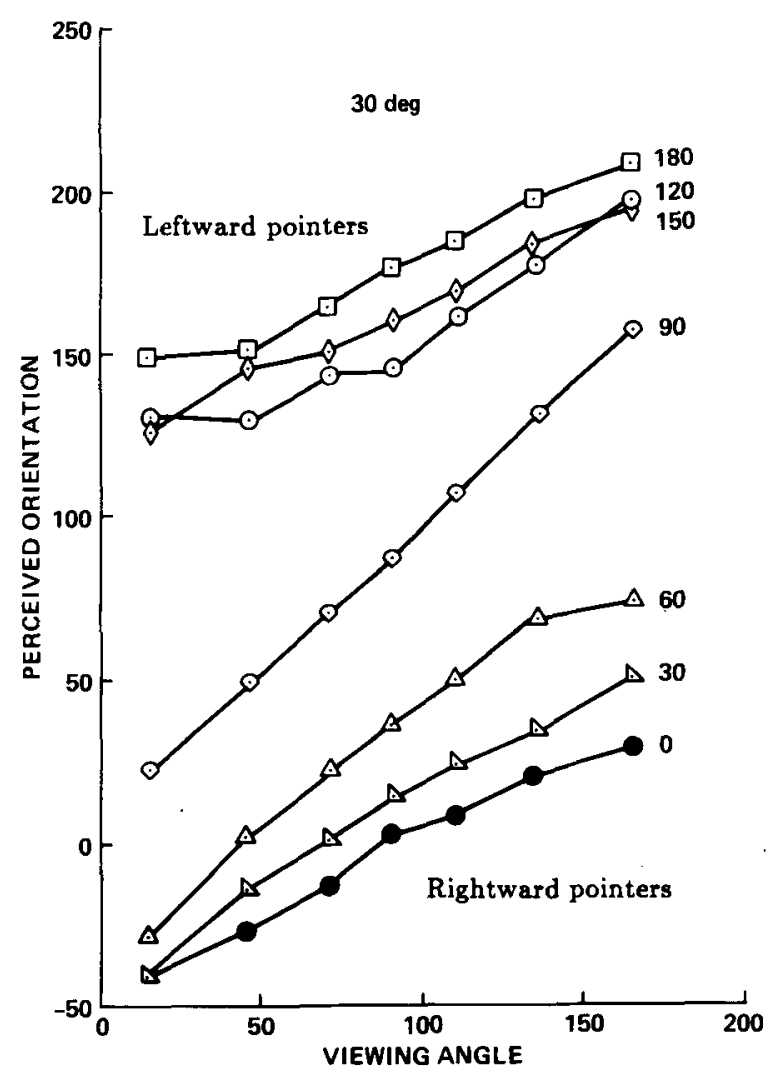

Figure 5. Mean perceived pointer orientation for viewing angles and depicted angles in Experiment 2 with a $30^{\circ}$ elevation condition. Plotted using Goldstein's conventions.

order to present each subject with the $98(7 \times 7 \times 2)$ distinct conditions.

Subjects. A new set of $\mathbf{8}$ paid subjects participated in the experiment. They were selected in the same manner and from the same subject pool as was used for the first experiment.

\section{Results}

The results of the second experiment were analyzed in a manner similar to that used in the first experiment. The interaction between viewing angle and depicted angle was again significant $[F(36,252)=8.194, p<.001]$. The interaction between viewing angle, depicted angle, and elevation angle was also significant $[F(36,252)=2.468$, $p<.001]$, but because the $\omega^{2}$ (Hays, 1973, pp. 512514 ), which measures the size of the effect, was so small $(0.002)$, the difference between elevation and depression of the pointer was omitted from further analysis.

The results, which are plotted in Figure 5, show the decreasing slope as the depicted angle of the pointer moves away from $90^{\circ}$. As the depicted angle changes from $0^{\circ}$ to $180^{\circ}$ in $30^{\circ}$ increments, the slopes of the perceived orientations shown in Figure 5 are, respectively, 0.62, $0.67,0.80,0.90,0.42,0.49$, and 0.43 . This change in slope corresponds to the differential rotation effect.

The results of Experiments 1 and 2 were combined to examine the effect of the elevation angle $-0^{\circ}$ or $30^{\circ}-$ on differential rotation. This was done by analyzing the data 


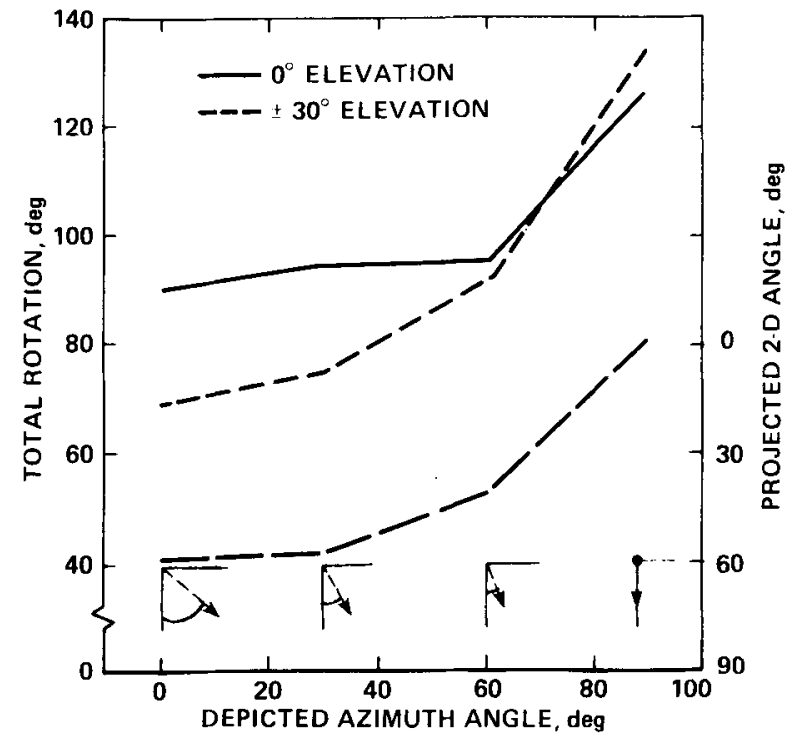

Figure 6. Comparison of total rotation measured in Experiments 1 and 2, also plotting the projected angle for Experiment 2. Total rotation is shown to be reduced for the larger projected angles. The angles shown by the arcs in the lower part of the figure are graphic representations of the projected two-dimensional angle of the pointers on the picture plane as seen by the subjects.

from both groups in a single ANOVA, with elevation of the pointer being treated as a between-groups condition. The resulting analysis showed that the depicted angle $x$ viewing angle $\times$ elevation angle interaction was significant $[F(36,504)=6.188, p<.001]$. Figure 6 shows one way, previously used by Goldstein (1979), of plotting an aspect of this interaction. The figure shows the total rotation that each depicted pointer underwent as the viewing angle was rotated $180^{\circ}$. It also shows that the introduction of the elevation angle to the pointer reduces the amount of total rotation, particularly for the depicted azimuth angles that are aligned with the plane of the photograph.

\section{GENERAL DISCUSSION}

The comparison of the results of Experiment 1 and Experiment 2 shows that changes in total rotation do not seem well correlated with the projected length of the pointer, which was the initial hypothesis. The essentially equivalent amount of total rotation for a $90^{\circ}$ depicted angle with either $0^{\circ}$ or $30^{\circ}$ elevation is particularly inconsistent with the initial hypothesis.

A general alternative explanation for the apparent position of the pointer is that introduction of an elevation angle increases the ambiguity regarding the true threedimensional position of the pointer. The increased ambiguity arises because the projected position of the pointer is now a function of two variables, as shown by Equation 12 in Appendix A. To the extent that the subjects are unable to precisely guess the amount of elevation, the true three-dimensional orientation is indeterminate. According to this view, when asked to judge the direction of the pointer, the subjects judge it to be in the plane of the photograph. This interpretation is the only one for which there is clear binocular evidence. The problem with this explanation, however, is that it does not explain why the subjects" "regression" to the plane of the photograph should be different for different amounts of azimuthal rotation of the pointer. Subsidiary assumptions would be required to rationalize this observed differential rotation. Without these assumptions, it would seem reasonable to expect the degree of ambiguity to be the same for all azimuth angles that resulted in a resolvable projected angle between the pointer and its supporting post.

There are two more specific alternative explanations that focus on ways the subjects can infer the actual position of the projected image in the plane of the photograph and thus discount its rotation. One method is based on the projected horizontal component of its image, which would provide the possibly important lateral stereo and convergence cues to the rotation of the picture out of the frontal plane. The projected horizontal component can be determined from the geometry in Appendix A. In the case of a pointer of length $l$, this projected length for a projection from distance $D$ with azimuth $\alpha$ and elevation $\epsilon$ is $l_{x}^{\prime}$ :

$$
l_{x}^{\prime}=\frac{l \cos (\alpha)}{D-l \sin (\alpha)} .
$$

The data, however, are also inconsistent with this explanation, because for a $0^{\circ}$ depicted azimuth angle, the horizontal component of the pointer is less for the $30^{\circ}$ elevation condition than for the $0^{\circ}$ elevation condition. Thus, one would expect more total rotation for the $30^{\circ}$ elevation condition, since the shorter horizontal projection of the pointer would provide less information regarding the position of its image on the plane of the photograph. However, the reverse is true: The $30^{\circ}$ elevation condition has less total rotation. Yet another analysis must be sought.

A second specific way to determine the orientation of the pointer in the picture plane is based on the ratio of the projected two-dimensional horizontal distance to the projected two-dimensional vertical distance. This ratio may also be thought of as the tangent of the projected angle $\delta^{\prime}$ on the surface of the picture, or the angle itself. Considered as an angle, its equation for a given elevation $\epsilon$ will be different for each depicted azimuth angle $\alpha$ according to the following equation:

$$
\delta^{\prime}=\arctan \left[\frac{\tan (\epsilon)}{\cos (\alpha)}\right] .
$$

The viewer's frontal projection $\left(\delta^{\prime \prime}\right)$ of this depicted elevation and azimuth angle in turn varies as a similar function of the viewing angle $v$ according to the following equation:

$$
\delta^{\prime \prime}=\arctan \left[\frac{\tan (\delta)}{\cos (v)}\right]
$$

The introduction of the elevation or depression angle into the pointer's orientation thus produces a two-dimensional 
angle on the surface of the photograph. The projection of the angle onto the observer's frontal plane is modulated by his viewing angle. The plot of this modulated two-dimensional angle in Figure 6 shows it to be correlated with the difference in total rotation measured between the $0^{\circ}$ and $30^{\circ}$ elevation conditions. In other words, as the two-dimensional angle increases, the difference in total rotation between the $0^{\circ}$ and $30^{\circ}$ elevation cases also increases.

One way in which the projected angle can modulate total rotation is by providing a variable amount of information concerning the rotational position of the picture surface. The larger the projected angle $d^{\prime}$, the greater the amplitude of the modulation of its secondary projection $d^{\prime \prime}$ by rotation $v$ of the photograph. As this modulation depth increases, the viewer can estimate more accurately the true rotation of the image of the pointer in the photograph. And as the viewer's estimate of the spatial position of this image improves, his estimate of the true depicted position of the pointer, which must discount the rotation of the image and the photograph, also improves.

One of the most prominent features of the total rotation curves that we measured in the present experiments is their curvilinearity. In this respect our measurements differ from Goldstein's (1979) plots of total rotation, which, although they show hints of the curvilinearity we found, generally show considerably straighter plots. Our total rotation curves are much more like Goldstein's curves for his shorter pointers, which were concave and downward, like ours. Our total rotation functions assume the shape that would be expected if the total rotation were a nonlinear function of the projected angle, $d^{\prime}$, which for a constant elevation is itself a function of the $\cos (\alpha)$; for example, Equation 5.

The finding that a projected angle is an important psychophysical variable influencing the interpretation of a virtual space from a perspective projection is consistent with the interpretation of two-dimensional projections of three-dimensional information. First, it concurs with the observation, elaborated on by Pirenne (1970), that circles and spheres are notoriously difficult to depict accurately in a picture. Correct projections of these objects almost always appear to be distorted, and artists commonly draw them to appear more circular than they should be based on a perspective projection. While these objects do have horizontal and vertical projections on the frontal plane that vary appropriately, they lack projected angles. Perhaps the problem with their realistic depiction can be attributed to this lack.

Second, the important psychophysical role for projected angles in determining perceived slant has been demonstrated by Attneave (1972; Attneave \& Frost, 1969; also see Perrone, 1982, for discussion of picture plane processing for determination of slant), who showed that the perceived slant of cubes can be predicted from the projected angles of their faces. Most significantly, Attneave showed that this determination of three-dimensional position of an object from its two-dimensional projection is dependent upon implicit assumptions about the three- dimensional properties of the actual object, such as the assumption that lines meet at right angles. Thus, in the absence of direct ranging information such as that provided by stereopsis or motion parallax, the fundamental ambiguity of a perspective projection must remain. The projected angles themselves cannot be used to specify the three-dimensional position of an object. The interpretation of the angles must be assisted by implicit assumptions concerning the three-dimensional structure they represent (Negrin \& Grunwald, 1985). In our experiments, the necessary assumptions concerning the depicted elevation were made possible by exposing the subjects to various stimuli during training. Additional exposure during the course of the experiment to $0^{\circ}$ and $180^{\circ}$ azimuth conditions showed the elevation angle itself. Without this exposure, the subjects' judgments of direction would have been computationally impossible, although the subjects might have been able to make some ad hoc assumptions if forced to do so.

Our measurements support the theory that, when available, the projected angle is the important psychophysical variable. However, in the case of a pointer with a $0^{\circ}$ elevation, there is no projected angle that varies as does a corresponding angle in the $30^{\circ}$ elevation case. Appendix B presents a more general formulation using a psychophysical variable that can apply to the geometry of both the $0^{\circ}$ and $30^{\circ}$ elevation case.

The interpretation that the elevated or depressed pointer reduces the amount of apparent rotation by improving the observer's ability to see the actual rotation of the picture plane is also consistent with classical reports concerning the kinetic depth effect (Wallach \& O'Connell, 1953). These reports show that a line varying in both length and direction is much more likely to appear to rotate in depth than is a line that varies only in length. Under the viewing conditions of the present experiments, the depressed/ elevated pointer provides just this joint cue to the picture rotation.

In our analysis, improved detection of picture plane rotation reduces the apparent rotation of the depicted pointer by providing the observer with information useful to discount the rotation. Conversely, one would expect changes that decrease the observer's ability to detect the picture plane orientation to increase the amount of apparent rotation. Recent reports in which the picture plane information was impaired by reversing the contrast of the stimuli (Goldstein, 1987, using white lines on a dark background) support this analysis. In this study the amount of apparent rotation was increased in comparison with the original one.

Deeper and quantitative application of this new measure of projected angle size, however, requires considerations of nonlinearities in the techniques used to record the observer's perceived angles. The placement of the pointer with respect to the observer's eyes and the photograph is critical in this respect, because the observer's perception of the pointer itself is based on a perspective projection. Existing psychophysical techniques to remove measurement nonlinearities should be adapted for this pur- 
pose and also to establish a standardized pointer placement (Blakemore \& Nachmias, 1971; Ellis, 1977). The resulting data would be more comparable between laboratories and more amenable to mathematical modeling.

An understanding of how observers judge the apparent direction of pointers coming out of the picture plane is not merely of academic interest. The geometric conditions and subjective judgments used in the experiments reported here closely resemble those associated with the use of the remote manipulator arm during approach of a spacecraft to a docking alignment target (NASA, 1985). A deeper understanding of how observers judge these geometrical relations when presented as a pictorial display should assist in the safe and efficient design of improved visual display aids for this task.

\section{REFERENCES}

Attneave, F. (1972). Representation of physical space. In A. W. Melton \& E. Martin (Eds.), Cognitive processes in human memory. New York: Wiley.

Attneave, F., \& Frost, R. (1969). The determination of perceived tridimensional orientation by minimum criteria. Perception \& Psychophysics, 6, 391-396.

Bengston, J. K., Stergios, J. C., Ward, J. L., \& Jester, R. E. (1980). Optic array determinants of apparent distance and size in pictures. Joumal of Experimental Psychology: Human Perception \& Performance, 6, 751-759.

Blakemore, C., \& Nachmias, J. (1971). The orientation specificity of two visual aftereffects. Journal of Physiology, 213, 157-174.

ElLIS, S. R. (1977). Orientation selectivity of the McCollough effect: Analysis by equivalent contrast transformation. Perception \& Psychophysics, 22, 534-544.

Foley, J. D., \& VAN DAM, A. (1982). Fundamentals of interactive computer graphics. London: Addison-Wesley.

GolDsteIN, E. B. (1979). Rotation of objects in pictures viewed at an angle: Evidence for different properties of two types of pictorial space. Journal of Experimental Psychology: Human Perception \& Performance, 5, 78-87.

GolDsteIN, E. B. (1987). Spatial layout, orientation relative to the observer, and perceived projection in pictures viewed at an angle. Journal of Experimental Psychology: Human Perception \& Performance, 13, 256-266.

HaYs, W. L. (1973). Statistics for the social sciences (2nd ed.). New York: Holt, Rinehart \& Winston.

McGreevy, M. W., \& Ellis, S. R. (in press). The effect of perspective geometry on judged direction in spatial information instruments. Human Factors.

National Aeronautics and Space Administration (1985). Orbital maneuvering vehicle: Preliminary definition study (internal note). Huntsville, AL: NASA Marshall Space Flight Center.

Negrin, M. \& Grunwald, A. (1985). A model for human spatial orientation using familiar object cues. Proceedings of the 5th European Annual Conference on Manual Control, Berlin: Free University of Berlin.

Perrone, J. (1982). Visual slant underestimation: A general model. Perception, 11, 641-654.

Pirenne, M. H. (1970). Optics, painting, and photography. Cambridge, England: Cambridge University Press.

Pirenne, H. H. (1975). Vision and art. In E. C. Carterette \& M. P. Friedman (Eds.), Handbook of perception: Vol. 5. Seeing (pp. 434488). New York: Academic Press.

RosinSKI, R. R., \& FARBER, J. (1980). Compensation for viewing point in the perception of pictured space. In M. A. Hagen (Ed.), The perception of pictures (Vol. 1, p. 137-76). New York: Academic Press.

Rosinski, R. R., Mulholland, T., Degelman, D., \& Farber, J.
(1980). Picture perception: An analysis of compensation. Perception \& Psychophysics, 28, 512-526.

Wallach, H. (1985, May). Perceiving a stable environment. Scientific American, 252, 118-124.

Wallach, H., \& O'ConNell, D. N. (1953). The kinetic depth effect. Journal of Experimental Psychology, 45, 205-217.

Wallach, H., Stanton, L., \& Becker, D. (1974). The compensation for movement-produced changes in object orientation. Perception \& Psychophysics, 15, 339-343.

\section{APPENDIX A}

In general terms, a perspective projection can be described by a multiplication of several matrices defining a linear transformation on a three-dimensional vector space of world coordinates that takes them into a two-dimensional vector space of a display surface. This process involves, sequentially, rotation, translation, scaling, and projection of the points defined in world coordinates; the process is clearly described in detail in computer graphics texts (e.g., Foley \& Van Dam, 1982, pp. 245-264).

Several aspects of the geometry used to view and construct the stimuli used in our experiments allow the development of relatively simpler expressions for some geometric properties of perspective projections that are important for this article. These expressions are based on the geometry illustrated in Figure 7, although they may arise from repetitive use of the projection described by the figure. In this figure we determine the perspective projection centered at $c$ of a line with length $l$ originating at reference point $r$. The line may be thought of as originating from the origin of a right-handed world coordinate system with $y$ up and $x$ to the right. In this frame of reference, the line makes an elevation angle $\epsilon$ with the $y$-axis and azimuth angle $\alpha$ with the $x$-axis. The projection is onto the frontal picture plane, which is orthogonal to the viewing direction and located $d$ units from the center of projection $c$, which is $D$ units from the reference point $r$.

The projection is accomplished by first projecting the line onto the $x z$ plane and then reprojecting this projection onto the $x$ - and $z$-axes, respectively. The $y$ projection is found directly from $\epsilon$. The lengths of the projections are determined by the magnification ratio $m$ :

$$
m=\frac{D-l \cos (\epsilon) \sin (\alpha)}{d}
$$

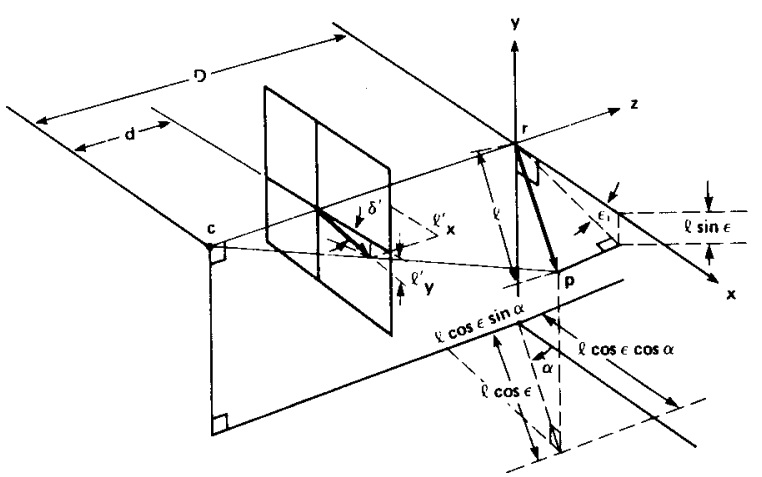

Figure 7. Projective geometry for the projection of a vector in three-dimensional space onto a two-dimensional picture plane. 
The ratio $m$ scales the horizontal and vertical projections onto the picture plane of the projections onto the $x$ - and $y$-axes by the following equations:

$$
l_{x}^{\prime}=\frac{l}{m} \cos (\epsilon) \cos (\alpha)
$$

and

$$
l_{y}^{\prime}=\frac{l}{m} \sin (\epsilon)
$$

The projected length of the pointer on the surface, $l_{s}^{\prime}$, arises from the usual Euclidean distance formula based on the projected $x$ and $y$ lengths of the pointer:

$$
l_{s}^{\prime}=\frac{l}{m} \sqrt{\left(\sin (\epsilon)^{2}+(\cos (\epsilon) \cos (\alpha))^{2}\right.} .
$$

When $d=1$, the resulting projection corresponds to that for determining lines of sight to an object $D$ units from a viewer. If both $d$ and $D$ are very large, $m$ approaches 1 and the projection corresponds to the orthogonal case. In any case, however, the projected angle $\delta^{\prime}$ may be directly determined from the ratio of the $x$ and $y$ projections, $l_{x}^{\prime}$ and $l_{y}^{\prime}$, of the pointer, because

$$
\tan \left(\delta^{\prime}\right)=\frac{l_{y}^{\prime}}{l_{x}^{\prime}}=\frac{l \sin (\epsilon)}{l \cos (\epsilon) \cos (\alpha)},
$$

so

$$
\tan (\delta)=\frac{\tan (\epsilon)}{\cos (\alpha)}
$$

\section{APPENDIX B}

One way to find a psychophysical variable that can apply to conditions used in both experiments is to express the amount of total rotation $\left(R_{t}\right)$ as a function of the norm of the cross-product of the two vectors on the surface of the photograph, $\mathbf{a}$ and $\mathbf{b}$, which are the arms of the projected angle. That is, $|\mathbf{a}|$ is the length of the pointer and $|\mathbf{b}|$ is the length from the pivot point to the point where the tripod legs attach. The length of $b$ is somewhat arbitrary but it is at least constant for both the $0^{\circ}$ and $30^{\circ}$ cases, so

$$
R_{t}=k|\mathbf{a} \times \mathbf{b}| \text {. }
$$

This cross-product exists for both the $0^{\circ}$ and $30^{\circ}$ cases and its norm equals $|\mathbf{a}||\mathbf{b}| \sin \left(\delta^{\prime}\right)$. In the special case of $0^{\circ}$ elevation, $\delta^{\prime}=90^{\circ}$ and $|\mathbf{a}|=(l / m) \cos (\alpha)$, so $R_{t}::[1 / \cos (\alpha)]$, which is Equation 1 discussed in the introduction. The value $|\mathbf{a} \times \mathbf{b}|$ generally correlates with the variation in apparent rotation for the two conditions, though the proportionality constant may be different for both conditions. The norm of the cross-product is particularly appropriate as a measure of the projection of the depicted angle because it combines the size of the projected angle with the length of its arms in a natural and mathematically tractable way. It is additionally attractive because it has a simple geometrical interpretation, that is, the area of the parallelogram whose sides are $\mathbf{a}$ and $\mathbf{b}$.

(Manuscript received June 27, 1986; revision accepted for publication May 28, 1987.) 